\title{
The PhenoGen Informatics website: tools for analyses of complex
} traits

Sanjiv V Bhave ${ }^{1}$, Cheryl Hornbaker ${ }^{1}$, Tzu L Phang1 ${ }^{1}$, Laura Saba1, Razvan Lapadat ${ }^{1}$, Katherina Kechris',2, Jeanette Gaydos' ${ }^{1}$, Daniel McGoldrick ${ }^{1}$, Andrew Dolbey ${ }^{1}$, Sonia Leach ${ }^{1}$, Brian Soriano1, Allison Ellington ${ }^{1}$, Eric Ellington ${ }^{1}$, Kendra Jones ${ }^{1}$, Jonathan Mangion ${ }^{3}$, John K Belknap ${ }^{4}$, Robert W Williams ${ }^{5}$, Lawrence E Hunter ${ }^{1}$, Paula L Hoffman ${ }^{1}$ and Boris Tabakoff*1

Address: ${ }^{1}$ Department of Pharmacology, University of Colorado at Denver and Health Sciences Center, Aurora, CO 80045, USA, ${ }^{2}$ Department of Preventive Medicine and Biometrics, University of Colorado at Denver and Health Sciences Center, Aurora, CO 80045, USA, ${ }^{3} \mathrm{MRC}$ Clinical Sciences Centre, Faculty of Medicine, Imperial College, London W12 0NN, UK, ${ }^{4}$ US Department of Veterans Affairs Medical Center, Portland, Oregon 97239, USA and ${ }^{5}$ Department of Anatomy and Neurobiology, University of Tennessee Health Science Center, 855 Monroe Avenue, Memphis, TN 38163, USA

Email: SanjivV Bhave - sanjiv.bhave@uchsc.edu; Cheryl Hornbaker - cheryl.hornbaker@uchsc.edu; Tzu L Phang - tzu.phang@uchsc.edu; Laura Saba - laura.saba@uchsc.edu; Razvan Lapadat - razvan.lapadat@uchsc.edu; Katherina Kechris - katerina.kechris@uchsc.edu; Jeanette Gaydos - jeanette.gaydos@uchsc.edu; Daniel McGoldrick - djmcgoldrick@comcast.net; Andrew Dolbey - andrew.dolbey@uchsc.edu; Sonia Leach - sonia.leach@uchsc.edu; Brian Soriano - brian.soriano@uchsc.edu; Allison Ellington - allison.ellington@uchsc.edu; Eric Ellington - eric.ellington@uchsc.edu; Kendra Jones - kendra.jones@uchsc.edu; Jonathan Mangion - jon.mangion@csc.mrc.ac.uk; John K Belknap - belknajo@ohsu.edu; Robert W Williams - rwilliams@nb.utmem.edu; Lawrence E Hunter - larry.hunter@uchsc.edu; Paula L Hoffman - paula.hoffman@uchs.edu; Boris Tabakoff* - boris.tabakoff@uchsc.edu

* Corresponding author

Published: 30 August 2007

BMC Genetics 2007, 8:59 doi:10.1 186/147|-2156-8-59
Received: 16 December 2006

Accepted: 30 August 2007

This article is available from: http://www.biomedcentral.com/I47/-2/56/8/59

(C) 2007 Bhave et al; licensee BioMed Central Ltd.

This is an Open Access article distributed under the terms of the Creative Commons Attribution License (http://creativecommons.org/licenses/by/2.0), which permits unrestricted use, distribution, and reproduction in any medium, provided the original work is properly cited.

\begin{abstract}
Background: With the advent of "omics" (e.g. genomics, transcriptomics, proteomics and phenomics), studies can produce enormous amounts of data. Managing this diverse data and integrating with other biological data are major challenges for the bioinformatics community. Comprehensive new tools are needed to store, integrate and analyze the data efficiently.

Description: The PhenoGen Informatics website http://phenogen.uchsc.edu is a comprehensive toolbox for storing, analyzing and integrating microarray data and related genotype and phenotype data. The site is particularly suited for combining QTL and microarray data to search for "candidate" genes contributing to complex traits. In addition, the site allows, if desired by the investigators, sharing of the data. Investigators can conduct "in-silico" microarray experiments using their own and/or "shared" data.

Conclusion: The PhenoGen website provides access to tools that can be used for high-throughput data storage, analyses and interpretation of the results. Some of the advantages of the architecture of the website are that, in the future, the present set of tools can be adapted for the analyses of any type of highthroughput "omics" data, and that access to new tools, available in the public domain or developed at PhenoGen, can be easily provided.
\end{abstract}




\section{Background}

The need for data sharing in the context of "omics" data has been underscored in a number of recent articles $[1,2]$. Funding agencies have also emphasized the need and expectation of data sharing among scientists [3]. In particular, the National Institutes of Health (NIH) implemented a data sharing policy that requires that any applications for funding of $\$ 500,000$ or more to specifically indicate a plan for data sharing. Most "omics" studies reach well beyond this fiscal threshold.

The magnitude and complexity of "omics" data have fueled interactions and cooperation among multiple investigators and institutions. These interactions have led to the formation of a number of microarray-based gene expression databases [4-12], both within public and commercial domains, and the development of associated tools necessary for high-throughput data analysis (see Table 1). Some of these databases also aid in understanding the biological inferences of the results (Table 1). The PhenoGen toolbox was originally created to facilitate interactions within the INIA consortium of investigators. In brief, the goals and purpose of the INIA (Integrative Neuroscience Initiative on Alcoholism) consortium are to identify the molecular, cellular, and behavioral neuroadaptations that occur in the brain reward circuits associated with the extended amygdala and its connections as a result of exposure to ethanol. Although PhenoGen web tools were initially created for the consortium members, the integrated tools described here are now accessible to the global scientific community.

\section{Construction and content}

An example of the work-flow at PhenoGen is shown in Figure 1. We maintain a local copy of MIAMExpress to insure MIAME/MAGE compliance of the microarray data stored in our database. For normalization, filtering and/or statistical analyses we have used various packages available in "R". A brief demonstration of how investigators can utilize our site to search for "candidate genes" for a given complex trait, e.g., fear conditioning in mice, is provided in Additional file 1.

The PhenoGen website is currently constructed to allow data to be classified as "Semi-public" or "Open Access". All of the information about the data uploaded at the PhenoGen website is visible to every registered user. The total number of microarrays, under different categories, from which data are available on PhenoGen is given in Table 2. Registered users have full access to data that are classified as "Open Access" and do not need to obtain permission from the curator of the data. However, users cannot access or download the "Semi-public" data unless the curator of the data (the Principal Investigator) grants permission to do so. Registered users can use the data for "in-silico" experiments on the website or can download the data for use with their own statistical software. At PhenoGen the curator(s) of the data (the Principal Investigators) also have an option to submit the data to microarray data repositories, such as ArrayExpress, as required by a number of journals [see Additional file 2: pages 115 to 116 in PhenoGen user manual].

\section{Utility}

To perform an "in-silico" experiment, users can select arrays from the current database (see Table 2) residing on the site (arrays can only be included in an "in-silico" experiment if the user has been granted access to the data or the data are in the Open Access domain). Microarray data from different laboratories can be combined to form "insilico" experiments. Users can also upload microarray data, either Affymterix, CodeLink (Applied Microarrays, Inc., Tempe, AZ, now manufactures these arrays) or custom arrays (if chip definition files are provided), using MIAMExpress, for further analyses. A series of quality control steps can be carried out, once the user has selected arrays to perform the "in-silico" experiment. This should be done to ensure compatibility and overall quality of the arrays [see Additional file 2: pages 31-42 in PhenoGen user manual]. The data from arrays in an "in-silico" experiment can be normalized, filtered and statistically analyzed utilizing several normalization and statistical procedures available on-site. At numerous points in this process the user can download data, raw or normalized, from experiments being performed on site, for use with other statistical packages of his/her choice.

As with some other databases, PhenoGen offers a range of options for microarray data normalization, filtering and statistical analyses, including corrections for multiple comparisons. Users can compare gene expression profiles in two groups using any one of the available options, or can use one-way or two-way ANOVA models to check for overall differences when comparing more than two groups. We plan to provide tools to carry out clustering (kmeans and hierarchical) analyses of microarray data in the near future. Furthermore, once an "in silico" experiment has been created, and the microarray data normalized, the user can search the database to determine the expression levels of any particular transcript(s). After choosing the correct (created) experiment, the user enters the probeset ID, or gene name or symbol (or any other annotation ID from the most popular genomic databases) [see Additional file 2: pages 60, 76-79 in PhenoGen user manual] and clicks "search", leading to display of expression data for the gene or genes in the chosen experiment. These data can be downloaded.

In addition to the standard statistical tools for assessing differential gene expression between or among groups, 
Table I: Comparison of microarray databases (and associated tools) in public domain

\begin{tabular}{|c|c|c|c|c|c|c|}
\hline Functionality & Array Express & GEO & $\begin{array}{l}\text { GeneNetwork/ } \\
\text { WebQTL }\end{array}$ & $\begin{array}{c}\text { Stanford } \\
\text { Microarray } \\
\text { database }\end{array}$ & BASE & PhenoGen \\
\hline $\begin{array}{l}\text { Data warehouse } \\
\text { (MIAME } \\
\text { compliant) }\end{array}$ & Yes & Yes & No & Yes & Yes & Yes \\
\hline \multicolumn{7}{|l|}{$\begin{array}{l}\text { Microarray } \\
\text { platform support }\end{array}$} \\
\hline Oligonucleotide & Yes & Yes & Yes & Yes & No & Yes \\
\hline cDNA & Yes & Yes & Yes & Yes & Yes & Yes \\
\hline \multicolumn{7}{|l|}{$\begin{array}{l}\text { Microarray data } \\
\text { search }\end{array}$} \\
\hline Gene based & Yes & Yes & Yes & Yes & Yes & No \\
\hline $\begin{array}{r}\text { Hybridization (array) } \\
\text { based }\end{array}$ & Yes & Yes & No & Yes & Yes & Yes \\
\hline Expt based & Yes & Yes & Yes & Yes & Yes & Yes \\
\hline Sample attributes & Yes & Yes & No & Yes & Yes & Yes \\
\hline \multicolumn{7}{|l|}{$\begin{array}{l}\text { Microarray data } \\
\text { retrieval }\end{array}$} \\
\hline Gene based & Yes & Yes & Yes & Yes & Yes & Yes \\
\hline $\begin{array}{r}\text { Hybridization (array) } \\
\text { based }\end{array}$ & Yes & Yes & No & Yes & Yes & Yes \\
\hline Expt based & Yes & Yes & No & Yes & Yes & Yes \\
\hline \multicolumn{7}{|l|}{$\begin{array}{l}\text { Microarray data } \\
\text { analysis }\end{array}$} \\
\hline QC & No & No & No & Yes & No & Yes \\
\hline Normalization & Yes & No & Yes & Yes & Yes & Yes \\
\hline Filtering & Yes & No & No & Yes & Yes & Yes \\
\hline \multicolumn{7}{|l|}{ "in-silico analysis" } \\
\hline Data sharing & Yes & No & ? & Yes & Yes & Yes \\
\hline $\begin{array}{r}\text { "in-silico } \\
\text { experiments" }\end{array}$ & Yes & No & No & $?$ & $?$ & Yes \\
\hline Statistics & & & "Different options" & & "Plug-Ins" & \\
\hline Basic stats & Yes & No & Yes & Yes & $?$ & Yes \\
\hline ANOVA & Yes & No & No & Yes & $?$ & Yes \\
\hline Clustering & Yes & No & Yes & Yes & $?$ & No \\
\hline Correlation & No & No & Yes & No & $?$ & Yes \\
\hline \multicolumn{7}{|l|}{ Gene lists } \\
\hline $\begin{array}{r}\text { Annotations } \\
\text { (Dynamic) }\end{array}$ & No & No & Yes & Yes & No & Yes \\
\hline Comparisons & No & No & Yes & $?$ & No & Yes \\
\hline eQTL & No & No & Yes & No & No & Yes \\
\hline $\begin{array}{r}\mathrm{PQTL}-\text { Gene } \\
\text { location overlapp }\end{array}$ & No & No & No & No & No & Yes \\
\hline $\begin{array}{r}\mathrm{PQTL}-\mathrm{eQTL} \\
\text { overlapP }\end{array}$ & No & No & No & No & No & Yes \\
\hline Promoter analysis & No & No & No & No & No & Yes \\
\hline Literature search & No & No & No & No & No & Yes \\
\hline \multicolumn{7}{|l|}{$\begin{array}{l}\text { System } \\
\text { requirements }\end{array}$} \\
\hline Local & Yes & No & No & Yes & Yes & No \\
\hline Web-based & Yes & Yes & Yes & Yes & Yes & Yes \\
\hline \multicolumn{7}{|l|}{ LIMS } \\
\hline Built-in & Yes & No & No & Yes & Yes & No \\
\hline \multicolumn{7}{|l|}{$\begin{array}{l}\text { Programming } \\
\text { expertise needed }\end{array}$} \\
\hline Expert & & & & Yes & Yes & \\
\hline Novice & Yes & & & Yes & Yes & \\
\hline None & & Yes & Yes & & & Yes \\
\hline $\begin{array}{l}\text { Functionality index } \\
\text { (Yes/No ratio) }\end{array}$ & 2.333 & 0.666 & 0.933 & 3.83 & 1.888 & 6.500 \\
\hline
\end{tabular}

This table describes various microarray databases. The majority of the functions listed are described in detail in Additional file 2 (PhenoGen user manual). "System requirements" indicates whether there are special computational requirements to use the database and associated tools; "LIMS" (Laboratory Information Management System) indicates whether LIMS is available; and "Programming expertise needed" is self-explanatory. Functionality index is a ratio of the number of "Yeses" to "Nos" for a given database. "?" indicates that the functionality of the database was not readily evident ("?" were not used to determine functionality index). 


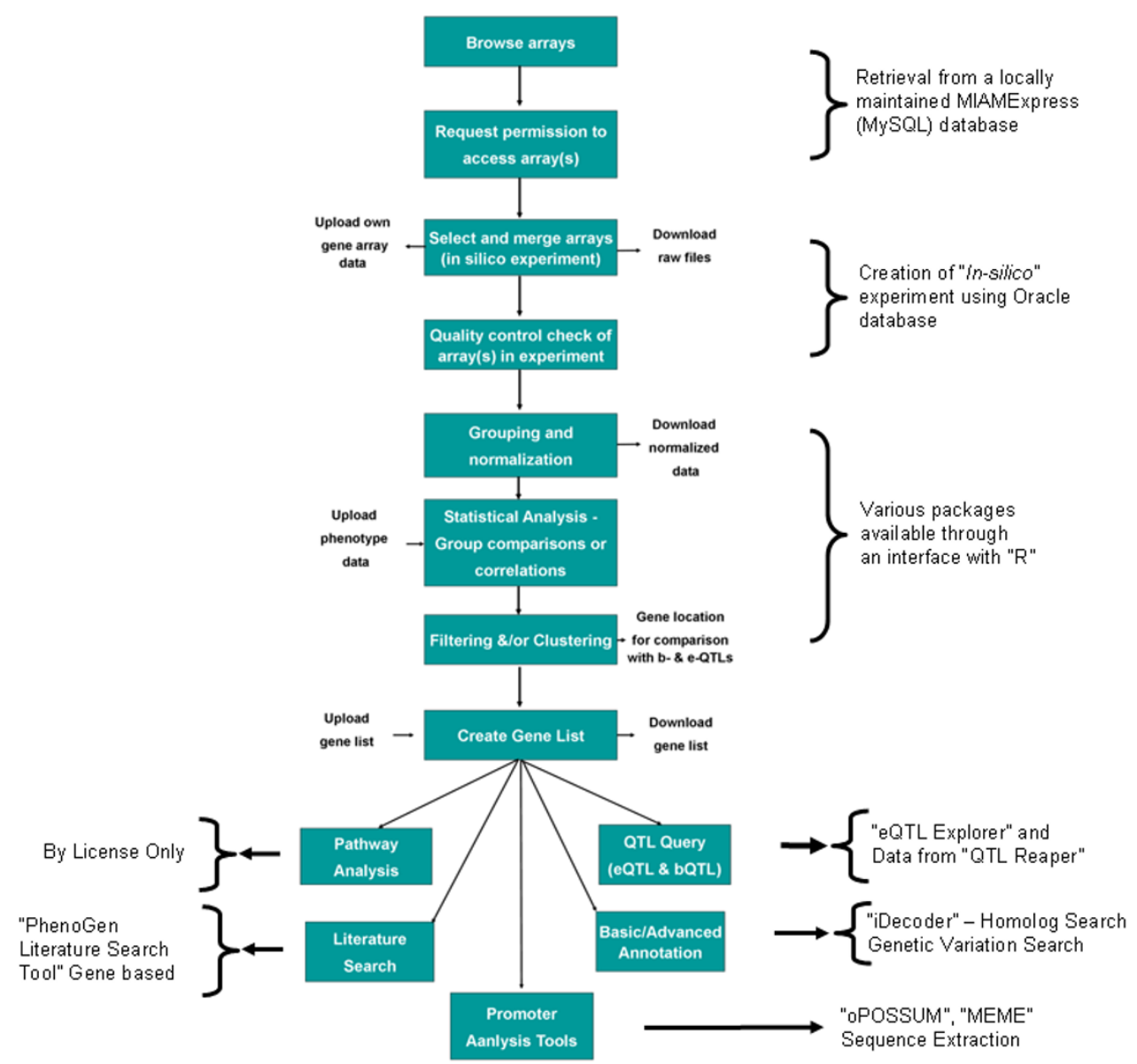

Figure I

The work-flow at PhenoGen. This flow chart demonstrates how the work-flow for analysis of data at the PhenoGen website can be organized and shows different programming languages and tools available at PhenoGen.

users can analyze the correlation between gene expression levels and phenotype (behavioral, biochemical or physiological). Such correlation analyses have been used in recent studies, by others [13-15] and our group [16], to ascertain "candidate genes" for complex traits with either a panel of recombinant inbred (RI) strains of mice (or rats) or a panel of inbred strains of mice. Users can access data available on site for whole brain gene expression profiles from either 20 inbred and 30 RI (BXD RI) strains of mice or 27 RI strains from the HXB/BXH panel of rats [17], and compare these data on gene expression to phenotypic data obtained with these same strains and species of animals in the user's laboratory, or to other phenotypic data (in the literature) related to brain function. Users can upload phenotype data (as a ".txt" file) for evaluating the correlation of gene expression with the phenotype. An example of such analysis of correlation of whole brain gene expression profiles with the contextual fear conditioning response in a panel of BXD RI strains, carried out using tools available on PhenoGen, is given in Additional file

Another distinguishing feature of PhenoGen is its multiple offerings for further data analysis, once a list of differentially expressed or correlated genes is generated on site or up-loaded de novo. Complex behavioral traits reflect variations in biochemistry, physiology, and anatomy that are determined by the action and interaction of several or many genes. We $[16,18]$, and others $[19,20]$ have indicated that the combined use of gene expression data together with QTL (quantitative trait locus) analysis can provide for a better understanding of the genetics of complex traits. The availability of techniques of genetic mapping and statistical analysis has allowed association of 
Table 2: Data available on PhenoGen

\begin{tabular}{|c|c|c|c|c|}
\hline \multicolumn{5}{|c|}{ Current array count } \\
\hline Organism & $\begin{array}{r}\text { Category/genetic } \\
\text { modification }\end{array}$ & $\begin{array}{c}\text { Open } \\
\text { access }\end{array}$ & $\begin{array}{l}\text { Requires } \\
\text { permission }\end{array}$ & Total \\
\hline Fly & & 0 & 24 & 24 \\
\hline Human & & 0 & 4 & 4 \\
\hline Mouse & & 557 & 142 & 699 \\
\hline & C57BL/6JxFVB/N FI & 12 & 0 & 12 \\
\hline & Gene knock out & 8 & 16 & 24 \\
\hline & Inbred strain & 229 & 78 & 307 \\
\hline & Knock down & 20 & 0 & 20 \\
\hline & None & 0 & 4 & 4 \\
\hline & $\begin{array}{r}\text { Recombinant inbred } \\
\text { strain }\end{array}$ & 168 & 0 & 168 \\
\hline & Selective breeding & 70 & 0 & 70 \\
\hline & Transgenic & 50 & 44 & 94 \\
\hline Rat & & 0 & 302 & 302 \\
\hline & Congenic strain & 0 & 10 & 10 \\
\hline & Inbred strain & 0 & 16 & 16 \\
\hline & None & 0 & 7 & 7 \\
\hline & $\begin{array}{r}\text { Recombinant inbred } \\
\text { strain }\end{array}$ & 0 & 146 & 146 \\
\hline & Selective breeding & 0 & 123 & 123 \\
\hline Total arrays & & 557 & 472 & 1029 \\
\hline
\end{tabular}

At present PhenoGen has microarray data from 1029 "samples" from different categories. Each "sample" represents mRNA obtained from an individual (animal, human or insect) sample and hybridized to an array (either Affymterix, CodeLink or a Custom oligonucleotide array). Data from any of these well-identified individual arrays can be used to conduct an "in-silico" experiment.

complex behavioral traits with genomic loci (QTL analysis). In short, QTLs are the genomic regions on the chromosomes that can explain a portion of the genetic variation within a given complex trait. Most complex traits are also significantly susceptible to environmental influences.

A premise of QTL analysis is that the genetic material that contributes to the variance in the trait of interest is located in the area of the genome defined by the QTL(s) for the trait. A number of different factors, such as polymorphism(s) in the coding or regulatory region(s) of gene(s), resulting in either a change in function and/or a change in expression (mRNA) of the gene(s), may contribute to a QTL. Therefore one can "filter" the differentially expressed genes in the brains of animals which differ significantly in the manifestation of the trait, or genes whose expression levels correlate with the magnitude of the trait of interest across multiple strains of animals, through a QTL filter. In other words, one can ascertain the genomic location of differentially expressed or correlated genes, and determine whether the location of these genes falls into QTLs determined for the trait of interest. Localization of differentially expressed or correlated genes within a QTL for a trait of interest adds significant weight to the supposition that the gene located within the QTL is one contributing to the variance in that trait. The PhenoGen website allows users to access information for gene location in the genomes of mouse, rat and human, to access data (MGI) on QTLs for a number of traits, and to analyze whether the location of genes falls within relevant phenotypic QTLs.

A major caveat to considering only the genes that have a physical location within behavioral or physiological QTLs as candidates for contributing to trait variance, is that the expression levels of genes that reside outside the behavio$\mathrm{ral} /$ physiologic QTLs may be regulated from within the behavioral/physiologic QTLs (an example of trans-regulation). The regulatory factor(s), themselves, would not have to be differentially expressed if a polymorphism resides in the target gene's expression regulatory region, and affects the function of the regulatory factor. Thus, any gene whose ultimate expression level is dependent on a genetic factor (cis or trans) within a behavioral/physiologic QTL becomes a candidate for contributing variance to the trait of interest.

We, and our colleagues, have used genomic marker data and information we have gathered on brain gene expression in male BXD RI mice and in HXB RI rats to determine the QTLs for the expression levels of genes in the brain (eQTLs). This e-QTL data, available on PhenoGen, allows for ascertainment of the genomic site of control of expression for a multitude of genes and allows for determination of whether the genes are cis- or trans-regulated.

In essence, the differentially expressed genes that reside within behavioral/physiologic QTLs, and have their expression regulated from within the same QTL (cis-regulated), and genes residing outside of the behavioral/physiologic QTLs, but whose expression is regulated from within a relevant behavioral/physiologic QTL (trans-regulated), could form the list of candidates contributing to a quantitative trait of interest. It has to be clear, however, that polymorphisms in the coding region of a gene can and do produce altered function of a gene product and can also significantly contribute to the trait of interest. Such polymorphisms, even when located in highly significant QTLs, would not be amenable to being identified by an analysis which relies on the premise that differential expression of a gene contributes to trait variance.

In addition to the "QTL Query tools", the PhenoGen website offers a wide variety of tools to "interpret" a gene list derived on site or up-loaded by the user. Such a list can include a few or hundreds of differentially expressed genes derived from a typical microarray experiment. At PhenoGen, users have access to tools, including annotation (basic and advanced), promoter analysis (to understand transcriptional regulation) and literature searches (including "co-citation" searches) for the entries in a list of differentially expressed genes. 
One of the ways to derive a "biological interpretation" of the results of the gene expression data is to analyze the biological annotations associated with the genes in a list of "candidates". i-Decoder, the underlying annotation tool used at PhenoGen, translates gene identifiers among many different nomenclatures, including gene symbols, RefSeq IDs, and probe names from both Affymetrix and CodeLink arrays, even when an up-loaded gene list contains multiple types of (non-identical) gene identifiers. This is accomplished by maintaining a local database of equivalents between identifiers that are available from the following eight sources: Affymetrix, GE Healthcare (formerly Amersham Biosciences), Ensembl, FlyBase, MGI, NCBI, RGD, and SwissProt [see Additional file 2]. In "Basic Annotation" tables every entry in the gene list is linked to the respective annotation in Entrez, MGI (or RGD), UniProt, and UCSC databases. A link is also provided to the "in-situ" hybridization images available at the Allen Brain Atlas to obtain regional distribution patterns of expression for genes in mouse brain. Another link is provided to the information at MGI about availability of genetically modified animals (transgenics, null mutants, etc.) for the genes in a gene list. Entries in the list of genes are also linked to information about genetic variations (e.g., single nucleotide polymorphism, insertion/deletion etc.), associated with the gene. In "Advanced Annotation Tables", users can personally select the available annotation information they wish to be displayed for the gene list.

To understand the transcriptional regulation of differentially expressed genes, users can use either oPOSSUM or MEME on the PhenoGen site. oPPOSUM uses humanmouse orthologs in calculating the over-representation of conserved transcription factor binding sites [21]. On the other hand, MEME explores the occurrences of previously uncharacterized transcriptional motifs [22]. Alternatively, the user can download the upstream sequences of genes of interest using the PhenoGen site and carry out similar analysis using other tools [23].

The literature search option on PhenoGen is an automated literature search that can be tailored to particular area(s) of interest by selecting a set of query terms. The automated literature search looks for articles in PubMed that mention any of the genes, including synonyms, in the gene list generated on site or uploaded by the user, and one or more of the chosen query terms. The results of the search are organized by the user-defined categories and by gene name, and contain direct links to PubMed citations. Also included in the results of a search is a list of articles where two or more of the genes from the gene list are cited in the same article (co-citation results). This allows the user to easily identify established relationships between genes.

\section{Discussion and conclusion}

The PhenoGen website consolidates many data analysis and interpretation tools in an easy point-and-click command format, and it can also facilitate the sharing of data between investigators across the globe. We have extensive and up-to-date transcriptome databases for whole brain gene expression for BXD RI and inbred strains of mice and RI rats that can be used in an "in-silico" analyses of correlation with phenotypes arising from the functions of the central nervous system, and to identify "candidate genes" using behavioral and expression QTL data. In addition to "QTL Tools" we provide a number of tools for promoter/ upstream sequence analysis, literature search, and tools to obtain annotation for a given list of genes. Although there are a number of other web-based tools available to carry out many of these analyses individually, PhenoGen provides one-stop access to most of these tools and to gene expression databases necessary to identify "candidate genes" for complex traits. Though at present the majority of the data available at PhenoGen is related to gene expression, the tools on the website can be adapted to handle other types of high throughput data, such as data derived from proteomic analysis. Another advantage of using the PhenoGen database, and associated analytical tools, is that users do not need expensive computational hardware and do not require extensive knowledge of programming languages. Websites such as PhenoGen are becoming indispensable for the life sciences research community so that scientists can arrive at biologically relevant interpretations of results of high-throughput studies[24].

\section{Availability and requirements}

The PhenoGen database and associated tools are available to the scientific community at http://pheno gen.uchsc.edu/. Users need to register in order to carry out any "in-silico" experiments using the data available in the open access component on the site or with their own data. Users can also conduct "in-silico" experiments using the data deposited in the semi-public component of our database by obtaining permission to do so from the investigators who deposited such data. Protocols and means for obtaining such permission are available on site.

\section{Authors' contributions}

SVB generated data from gene expression studies, assisted with e-QTL analysis, assisted in writing User Manual and this manuscript. CH performed coding and programming for all aspects of the PhenoGen website. TLP participated in organization of the website, and performed coding and testing. LS performed coding, statistical analysis testing, and helped in writing this manuscript. RL performed coding and testing, particularly in the e-QTL arena. KK performed coding and testing, particularly in transcription factor analysis tools. JG performed gene array analysis and 
site testing and assisted with the User Manual. DM performed coding for p-QTL analysis. AD performed coding for literature search modules. SL helped coding for statistical analysis modules in R. BS supervised and performed gene expression analysis and tested site elements. AE wrote User Manual. EE performed coding for integrating the PhenoGen with various public databases. KJ performed coding and assisted in programming for all aspects of the PhenoGen. JM developed of tools for e-QTL analyses (e-QTL Explorer). JKB introduced and helped program the QTL analysis in R. RWW introduced and assisted integration between WebQTL and PhenoGen. LEH supervised and directed coding and obtained funding.

PLH supervised collection of gene expression data, interpretation and testing the PhenoGen and helped with writing and editing this manuscript. BT conceived the underlying premises and structure for PhenoGen, organized the efforts of staff, secured funding and helped write and edit this manuscript

\section{Additional material}

\section{Additional file 1}

Example of use of PhenoGen website. This additional file provides an example of the use of the website to identify "candidate genes" for the contextual fear conditioning response in BXD RI mice.

Click here for file

[http://www.biomedcentral.com/content/supplementary/14712156-8-59-S1.pdf]

\section{Additional file 2}

PhenoGen user manual. This additional file is a user manual for the PhenoGen website.

Click here for file

[http://www.biomedcentral.com/content/supplementary/14712156-8-59-S2.pdf]

\section{Acknowledgements}

This work was supported in part by ROI AAI 3 I 62 (BT); U0I AA0I 3524 (INIA Project; LH; BT), and the Banbury Fund (BT).

\section{References}

I. Geschwind DH: Sharing gene expression data: an array of options. Nat Rev Neurosci 200I, 2:435-438.

2. Insel TR, Volkow ND, Li TK, Battey JF Jr., Landis SC: Neuroscience networks: data-sharing in an information age. PLoS Biol 2003, I:El7.

3. Baughman RW, Farkas R, Guzman M, Huerta MF: The National Institutes of Health Blueprint for Neuroscience Research. J Neurosci 2006, 26:10329-10331.

4. Kapushesky M, Kemmeren P, Culhane AC, Durinck S, Ihmels J, Korner C, Kull M, Torrente A, Sarkans U, Vilo J, Brazma A: Expression Profiler: next generation--an online platform for analysis of microarray data. Nucleic Acids Res 2004, 32:W465-70.

5. Brazma A, Kapushesky M, Parkinson H, Sarkans U, Shojatalab M: Data storage and analysis in ArrayExpress. Methods Enzymol 2006, $411: 370-386$.
6. Barrett T, Suzek TO, Troup DB, Wilhite SE, Ngau WC, Ledoux P, Rudnev D, Lash AE, Fujibuchi W, Edgar R: NCBI GEO: mining millions of expression profiles--database and tools. Nucleic Acids Res 2005, 33:D562-6.

7. Barrett T, Troup DB, Wilhite SE, Ledoux P, Rudnev D, Evangelista C, Kim IF, Soboleva A, Tomashevsky M, Edgar R: NCBI GEO: mining tens of millions of expression profiles--database and tools update. Nucleic Acids Res 2006, 35(Database issue):D760-D765.

8. Gollub J, Ball CA, Sherlock G: The Stanford Microarray Database: a user's guide. Methods Mol Biol 2006, 338: I9l-208.

9. Ball CA, Awad IA, Demeter J, Gollub J, Hebert JM, Hernandez-Boussard $T$, Jin $H$, Matese JC, Nitzberg M, Wymore F, Zachariah ZK, Brown PO, Sherlock G: The Stanford Microarray Database accommodates additional microarray platforms and data formats. Nucleic Acids Res 2005, 33:D580-2.

10. Troein C, Vallon-Christersson J, Saal LH: An introduction to BioArray Software Environment. Methods Enzymol 2006, 41 I:99- II9.

II. Saal LH, Troein C, Vallon-Christersson J, Gruvberger S, Borg A, Peterson C: BioArray Software Environment (BASE): a platform for comprehensive management and analysis of microarray data. Genome Biol 2002, 3:SOFTWARE0003.

12. Sherlock G, Ball CA: Storage and retrieval of microarray data and open source microarray database software. Mol Biotechnol 2005, 30:239-25I.

13. Hovatta I, Tennant RS, Helton R, Marr RA, Singer O, Redwine JM, Ellison JA, Schadt EE, Verma IM, Lockhart DJ, Barlow C: Glyoxalase I and glutathione reductase I regulate anxiety in mice. Nature 2005, 438:662-666

14. Korostynski M, Kaminska-Chowaniec D, Piechota M, Przewlocki R: Gene expression profiling in the striatum of inbred mouse strains with distinct opioid-related phenotypes. BMC Genomics 2006, 7: 146.

15. Nadler J], Zou F, Huang H, Moy SS, Lauder J, Crawley JN, Threadgill DW, Wright FA, Magnuson TR: Large-scale gene expression differences across brain regions and inbred strains correlate with a behavioral phenotype. Genetics 2006, I 74: I229-I236.

16. Saba L, Bhave SV, Grahame N, Bice P, Lapadat R, Belknap J, Hoffman $\mathrm{PL}$, Tabakoff $\mathrm{B}$ : Candidate genes and their regulatory elements: alcohol preference and tolerance. Mamm Genome 2006, I 7:669-688.

17. Pravenec M, Klir P, Kren V, Zicha J, Kunes J: An analysis of spontaneous hypertension in spontaneously hypertensive rats by means of new recombinant inbred strains. J Hypertens 1989 , 7:217-221.

18. Tabakoff B, Bhave SV, Hoffman PL: Selective breeding, quantitative trait locus analysis, and gene arrays identify candidate genes for complex drug-related behaviors. J Neurosci 2003, 23:4491-4498.

19. Schadt EE, Lamb J, Yang X, Zhu J, Edwards S, Guhathakurta D, Sieberts SK, Monks S, Reitman M, Zhang C, Lum PY, Leonardson A, Thieringer R, Metzger JM, Yang L, Castle J, Zhu H, Kash SF, Drake TA, Sachs A, Lusis AJ: An integrative genomics approach to infer causal associations between gene expression and disease. Nat Genet 2005, 37:710-717.

20. Mehrabian M, Allayee H, Stockton J, Lum PY, Drake TA, Castellani LW, Suh M, Armour C, Edwards S, Lamb J, Lusis AJ, Schadt EE: Integrating genotypic and expression data in a segregating mouse population to identify 5-lipoxygenase as a susceptibility gene for obesity and bone traits. Nat Genet 2005, 37: 1224-1233.

21. Ho Sui SJ, Mortimer JR, Arenillas DJ, Brumm J, Walsh CJ, Kennedy BP, Wasserman WW: oPOSSUM: identification of over-represented transcription factor binding sites in co-expressed genes. Nucleic Acids Res 2005, 33:3154-3164.

22. Bailey TL, Elkan C: The value of prior knowledge in discovering motifs with MEME. Proc Int Conf Intell Syst Mol Biol 1995, 3:2 1-29.

23. Tompa M, Li N, Bailey TL, Church GM, De Moor B, Eskin E, Favorov AV, Frith MC, Fu Y, Kent WJ, Makeev VJ, Mironov AA, Noble WS, Pavesi G, Pesole G, Regnier M, Simonis N, Sinha S, Thijs G, van Helden J, Vandenbogaert M, Weng Z, Workman C, Ye C, Zhu Z: Assessing computational tools for the discovery of transcription factor binding sites. Nat Biotechnol 2005, 23:|37-|44.

24. The database revolution. Nature 2007, 445:229 -2230 . 